\title{
THORACOSCOPIC REPAIR OF ESOPHAGEAL ATRESIA WITH DISTAL FISTULA IN A NEW BORN:
}

\author{
E. Brandigi, F. Molinaro, A.L. Bulotta, G. Di Maggio, R. Angotti, M. Messina \\ Division of Pediatric Surgery, Department of Medical sciences, surgery and neurosciences, \\ University of Siena, Siena, Italy
}

\begin{abstract}
Introduction. Esophageal atresia encompasses a group of congenital anomalies comprising of an interruption of the esophageal continuity with or without a persistent communication with the trachea. Esophageal atresia with tracheoesophageal fistula (type C) accounts for $85 \%$ of all esophageal atresia. Minimally invasive approach to correct esophageal atresia with distal fistula is becoming more generally accepted. The outcome of these technique are critically analyzed and compared with results from open repair. We present one case of type IIIB esophageal atresia treated by a thoracoscopic approach. Case Report. The patient was a 2-days-old infant male, weight $3 \mathrm{~kg}$ with esophageal atresia and distal tracheoesophageal fistula without other associated disease. A polidramanios was detected in prenatal age by a prenatal ultrasound evaluation. He underwent to a thoracoscopic repair of the defect. The operation was approached through the right chest using a three-trocar technique (three $5-\mathrm{mm}$ ) with the patient placed in a three-quarter prone position. The azygos vein was ligated by Ligature device. The fistula was ligated by two resorbable stiches suture and dissected, the proximal esophagus was opened and an anastomosis was made over a $6 \mathrm{Ch}$ nasogastric tube with interrupted and resorbable stiches suture. On the postoperative day 7, gastrografin swallow was performed and oral feeding was started. The patient's six month upper Gastrointestinal barium studies was normal. Discussion and conclusion. Thoracoscopic repair of esophageal atresia is considered to be one of the more advanced and most difficult pediatric surgical procedures and it undoubtely has necessary an elevated learning curve. The minimally invasive approach was larged accepted in the last ten years also for the well documented sequelae of traditional open repair of esophageal atresia. More experience is needed to determine the exact place of this approach.
\end{abstract}

Key words: esophageal atresia, thoracoscopy.

\section{INTRODUCTION}

Esophageal atresia (EA) encompasses a group of congenital anomalies comprising of an interruption of the continuity of the esophagus with or without a persistent communication with the trachea. EA with trachea-esophageal fistula(TEF) (type C) accounts for $85 \%$ of all EA. [1]

Traditionally, the repair of EA with TEF is ligation of fistula and primary anastomosis of both ends via open right thoracotomy. [2] The first reported thoracoscopic repair of EA was in 1999 and now, thoracoscopic approach is an effective alternative to the traditional approach to correct EA with distal fistula. [3]

We present one case of type IIIB esophageal atresia treated by a thoracoscopic approach.

\section{CASE REPORT}

A newborn male was transferred to us from another hospital with a clinical diagnosis of EA. He was born at 41 weeks with a birth weight of $3,1 \mathrm{~kg}$. Antenatally, polyhydramnios was detected and a nasogastric tube could not be passed after birth. X-ray confirmed the diagnosis and showed the end of the upper part of the esophagus at the T2-T3 level and the presence of gas in the bowel and stomach significative to distal TEF (Fig 1). Routine echocardiography showed the absence of cardiac malformation. No others associated anomalies were noted. On day 2 of life, the infant was taken for thoracoscopic repair of the anomaly. After induction of general anesthesia and nonselective orotracheal intubation, the patient was positioned in the left lateral decubitus position, slightly prone (FIg.2). Three 5-mm trocars were used. The first, was placed

Correspondence to:

Prof. Mario Messina

Chirurgia Pediatrica

Policlinico "Le Scotte", Viale Bracci 16, 53100 Siena, Italy

Tel.: +39.577.586501; Fax: +39.577.586174

E-mail: mario.messina@unisi.it 


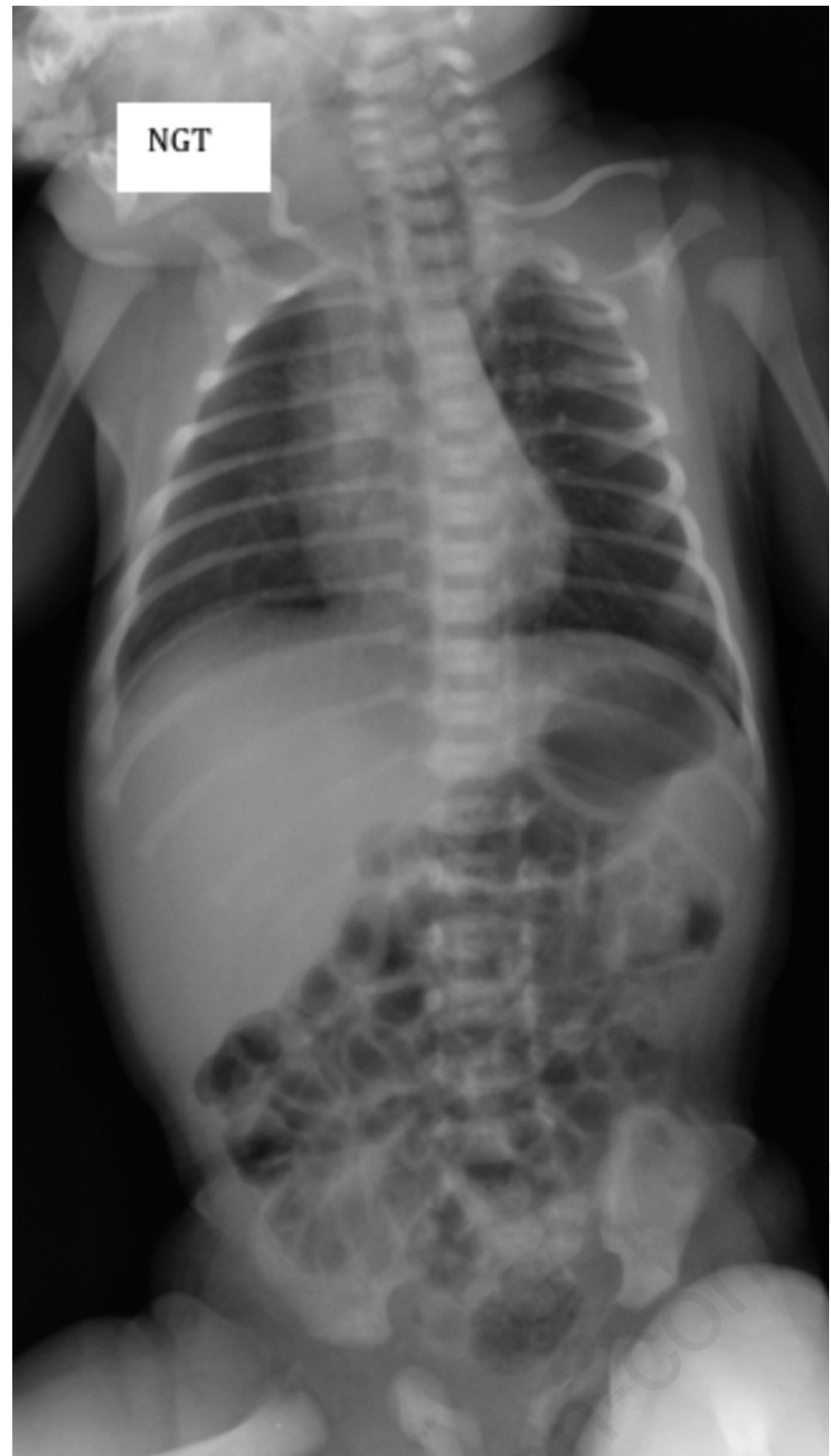

Figure 1. X-ray showed the presence of gas in the bowel.

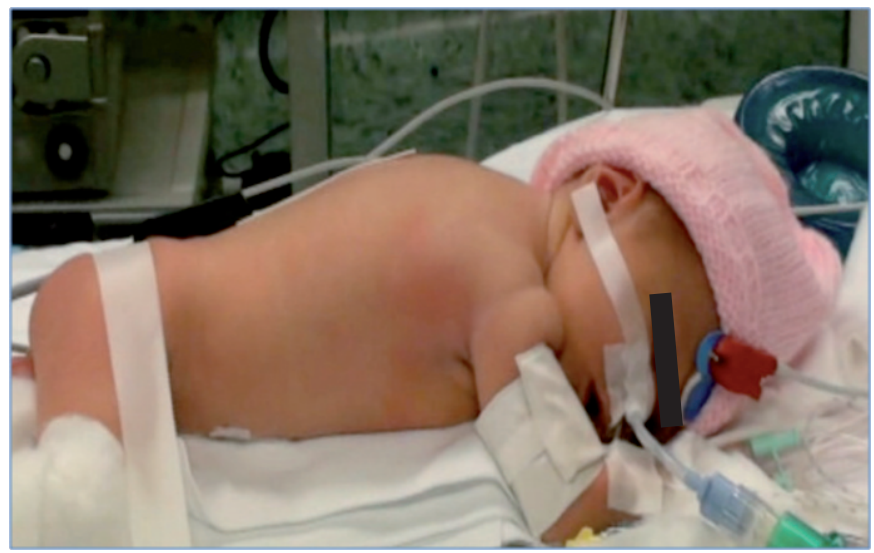

Figure 2. Surgical positioning. in the fifth intercostal space in the posterior axillary line and the pneumothorax was created $(0,5 \mathrm{l} / \mathrm{min}, 6$ mmhg). The second and third trocars were placed in the third and sixth intercostal spaces in the midaxillary line. The pleura overlying the posterosuperior mediastinum was incised, exposing the azygos vein. Access to the esophageal limbs and trachea was gained by division of the azygos vein using a $5-\mathrm{mm} \mathrm{Li}$ gasure. The distal esophageal pouch revealed a fistula connecting the distal pouch to the trachea(Fig.3). This fistula was ligated using a 4-0 Vicryl suture and subsequently dissected. The proximal esophageal pouch was then identified near $2 \mathrm{~cm}$ over the distal limb. The blind-ending pouch was opened and the back wall of the anastomosis was completed using multiple interrupted 4-0 Vicryl sutures tied intracorporeally (Fig.4). A $6 \mathrm{Ch}$ nasogastric tube was passed

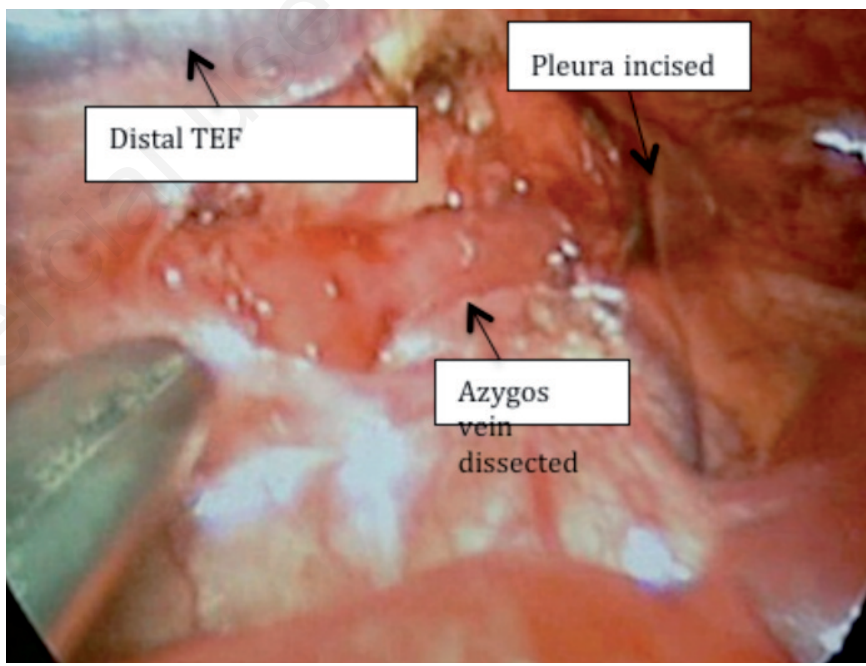

Figure 3. TEF riveled after Azygos vein dissection.

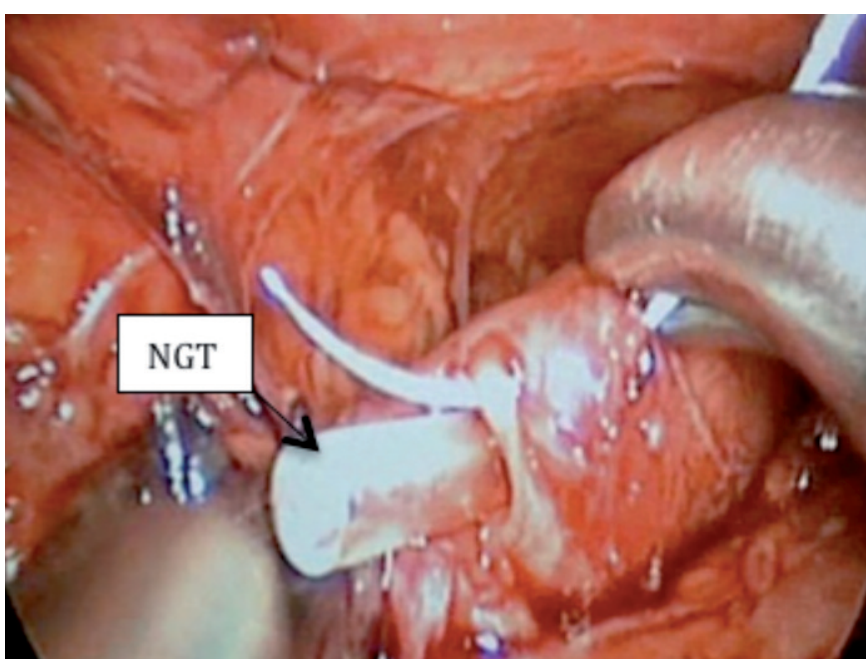

Figure 4. Nasogastric tube through the proximal pouch opened. 
nasally and directed through the anastomosis and the front wall of the anastomosis was then completed over the tube(Fig.5). Finally, a chest tube was left by the office of the sixth intercostal space in the posterior axillary line. The operating time was 240 minutes, and there were no intraoperative incidents.

The patient was transferred to the NICU postoperatively, he was extubated 1 week later, and esophageal contrast study was performed and showed no leak or stenosis (Fig.6). The baby made an excellent recovery and he was discharged on day 25 after the operation. The follow up was performed five months after surgery. A normal growth was showed and a upper Gastrointestinal barium study was negative.

\section{DISCUSSION AND CONCLUSIONS}

Thoracoscopic repair of esophageal atresia was large accepted in the last ten years.

The thoracoscopic approach gave the opportunity to avoid the injury to the chest wall and its consequences: the deformity of the chest wall, ribs fusion, scoliosis, muscle maldevelopment, and chronic pain. [4] Another advantage is excellent visualization of anatomic structures on the monitor with magnification. This improved visualization is especially true for identification of the tracheoesophageal fistula. [5]

However the 'technical hurdles are many' as pointed out by an acknowledged leader in thoracoscopic repairs, Steven Rothenberg. He describes the difficulties in suturing within such a small closed space as a major technical difficulty.[6] In fact, he evidently regards these technical demands as being so significantly challenging that he commented in a 2012 paper that 'for

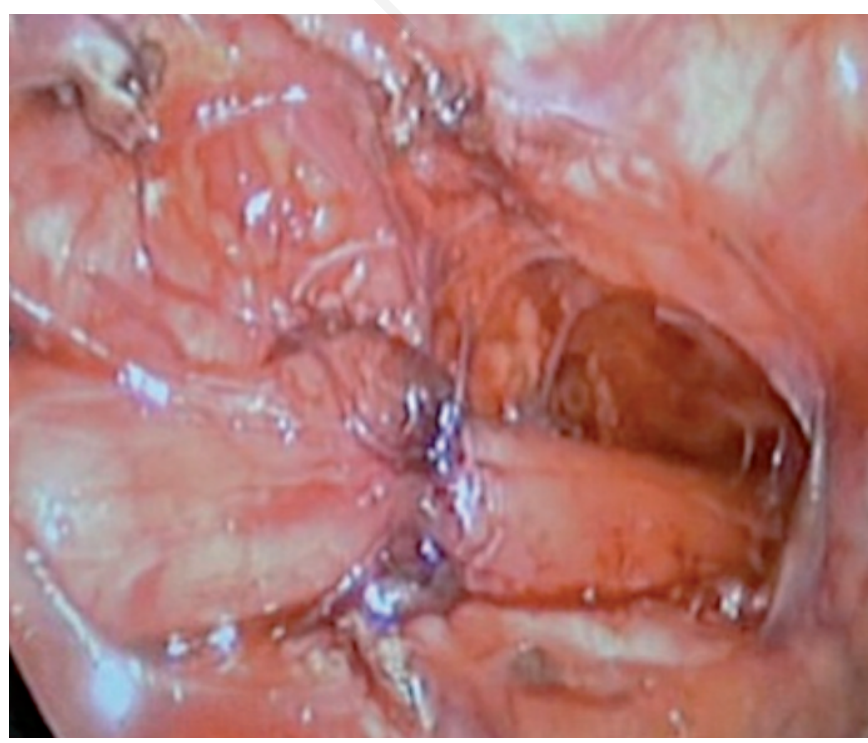

Figure 5. Completed anastomosis. this procedure to become more widely accepted, it may be necessary to develop a mechanical anastomotic device or self-knotting suture'. [7] The minimally invasive approach is considered to be one of advanced techniques and the most difficult pediatric surgical procedure. For all these reasons it undoubtedly has necessary an elevated learning curve. This operations must be accomplished by surgeons who have been performing advanced laparoscopic and thoracoscopic procedures in infants and children.

Also, some patients are probably not good candidates for the thoracoscopic approach. The operation is difficult in babies less than $2 \mathrm{~kg}$ and in babies with significant lung disease, since it requires the ipsilateral lung to be compressed with the operative pneumothorax to achieve an adequate working space. [2] On the other hand thoracotomy is a technically mastered and it is easier to teach. Several studies show no significant difference between two techniques in postoperative complications ( stricture and leak of the anastomosis) or in mortality. Holcomb et al performed a multi-centric study with 104 newborns affected by EA/TEF and treated with minimally invasive surgery. They conclude that their operative results are comparable to those achieved with the traditional operation.[2]

We choose the minimally invasive approach for the

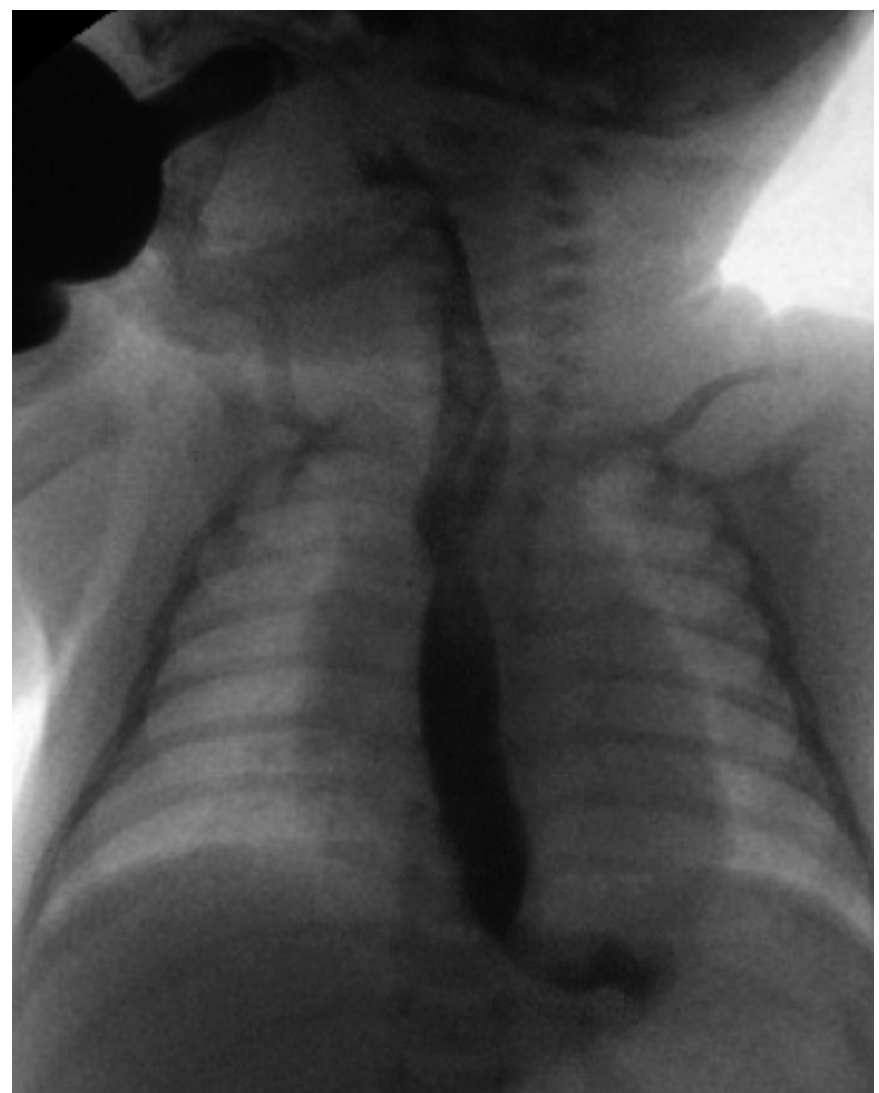

Figure 6. Contrast esophagogram on postoperative day 7 showing no leakage and good passage of contrast. 
condition of the patient and for our experience in laparoscopic and thoracoscopic procedures.

The most difficult part of the operation was the anastomosis and for this reason the time was longer than others reported in the literature. All the knots were tied intracorporeally, and we prefer this technique especially in newborns. Anastomosis was made tension free and the esophageal contrast study showed no complications.

In conclusion, there are many articles that report a comparison to two techniques, but there is not yet any evidences that thoracoscopic EA repair is better than open thoracotomy repair. [8]

There are advantages and disadvantages to both techniques as previously indicated.

We have revised the literature and the last one article (2011), showed that 'thoracoscopic repair of EA and TEF is better than open surgery'.(9)

While thoracoscopic repair is apparently safe and possibly advantageous in some respects when performed by a handful of experts, it should not be touted as the 'gold standard' to be utilized by all pediatric surgeons. [8]

We have used minimally invasive surgical techniques for many diseases into our experience.

Now, the main aim of anyone technique is a good anastomosis, survival, limited sequels and good quality of life [9].

\section{REFERENCES}

1. Demircan M, Aksoy T, Ceran C, Kafkasli A. Tracheal agenesis and esophageal atresia with proximal and distal bronchoesophageal fistulas. J Pediatr Surg 2008;43:e1-3.

2. Holcomb III GW, Rothenberg SS, Bax KM, et al. Thoracoscopic repair of esophageal atresia and tracheoesophageal fistula: a multiinstitutional analysis. Ann Surg 2005;242:422-8.

3. Lobe TE, Rothenberg SS, Waldschmidt J. Thoracoscopic repair of esophageal atresia in an infant: a surgical first. Pediatr Endosurg Innov Tech 1999;3:141-8.

4. Patkowsk D, Rysiakiewicz K, Jaworski W, et al. Thoracoscopic repair of tracheoesophageal fistula and esophageal atresia. J Laparoendosc Adv Surg Tech A 2009;19:S19-22.

5. Rothenberg SS. Thoracoscopic repair of tracheoesophageal fistula in newborns. J Pediatr Surg 2002;37:869-72.

6. Rothenberg SS. Thoracoscopic repair of esophageal atresia and tracheo-esophageal fistula. Semin Pediatr Surg 2005;14:2-7.

7. Rothenberg SS. Thoracoscopic repair of esophageal atresia and tracheo-esophageal fistula in neonates: evolution of a technique. $J \mathrm{La}$ paroendosc Adv Surg Tech A 2012;22:195-9.

8. Laberge JM, Blair GK. Thoracotomy for repair of esophageal atresia: not as bad as they want you to think! Dis Esophagus 2013;26:365-71.

9. Tovar JA, Fragoso AC. Current controversies in the surgical treatment of esophageal atresia. Scand J Surg 2011;100:273-8. 